\title{
The Symmetry and Efimov's Effect in Systems of Three-Quantum Particles
}

\author{
S. A. Vugal'ter ${ }^{1}$ and G. M. Zhislin ${ }^{2}$ \\ 1 Gorky State University, Gorky, USSR \\ 2 Radiophysical Research Institute, Gorky, USSR
}

\begin{abstract}
The finiteness of the discrete spectrum of three body Schrödinger operators restricted to certain symmetry subspaces is proved. The symmetry subspaces are those associated with nonzero angular momentum and those associated with two or three identical fermions.
\end{abstract}

\section{Introduction}

Investigation of discrete spectrum energy operators of three-particle quantum systems without bound subsystems showed that the discrete spectrum of such systems can be infinite even if the potentials between the particles decrease arbitrarily rapidly (Elfimov's effect). This possibility is realized (under some additional conditions) for three-particle systems when energy operators $h_{\alpha}$ of two or three two-particle subsystems have virtual levels $[1,2]$ (see also [3]).

The presence of the operator $h_{\alpha}$ virtual level in this situation is connected with the existence of such solution $\varphi$ of equation $h_{\alpha} \varphi=0$ so that $|\nabla \varphi(x)| \in \mathscr{L}_{2}\left(R^{3}\right)$, $\varphi(x) \notin \mathscr{L}_{2}\left(R^{3}\right)$.

This article presents an investigation of the discrete spectrum of three-particle operators in some symmetry spaces. It is proved that in these spaces Efimov's effect is absent ${ }^{1}$ so that for short-range potentials the discrete spectrum is finite.

Our proof of the finiteness is founded, mainly, on the investigation of virtual levels of two-particle Hamiltonians in the function subspaces from $\mathscr{L}_{2}\left(R^{3}\right) \ominus P^{(0)} \mathscr{L}_{2}\left(R^{3}\right)$ (see Sect. 3). It is established, in particular, (Theorem 3.1), that in these subspaces the presence of virtual levels of a two particle operator is connected with the presence of its zero eigenvalue as distinct from the case when the symmetry is not taken into account.

The main results of the work are Theorems 2.1-2.5 which are formulated in Sect. 2 and proved in Sect. 4. Theorem 3.1 is proved in Sect. 3. All auxiliary assertions and their proofs are in Sect. 5 .

1 In physics articles this assertion appeared earlier [4] but this fact was not proved mathematically rigorously 


\section{The Basic Results}

Let $Z_{1}$ be an arbitrary quantum system of 3-particles $x_{i}=\left(x^{(3 i-2)}, x^{(3 i-1)}, x^{(3 i)}\right)$ with masses $m_{i}, x=\left(x_{1}, x_{2}, x_{3}\right), H_{0}$ be the energy operator of system $Z_{1}$ written after invariant separation of center of mass motion and after introduction an inner product $(x, \tilde{x})_{1}=\sum_{i=1}^{3} m_{i}\left(x_{i}, \tilde{x}_{i}\right)$. It is easy to see that [6]

$$
H_{0}=-\frac{1}{2} \Delta_{0}+\sum_{(i, j), i<j}^{1,3} V_{i j}\left(\left|x_{i j}\right|\right),
$$

where $x_{i j}=x_{i}-x_{j}, \Delta_{0}-$ is the Laplacian on

$$
R_{0}=\left\{x \mid x=\left(x_{1}, x_{2}, x_{3}\right), \sum_{i=1}^{3} m_{i} x_{i}=0\right\} .
$$

As to $V_{i j}$ we shall assume that

$$
\begin{gathered}
V_{i j}^{*}=V_{i j}\left(\left|x_{1}\right|\right) \in \mathscr{L}_{2, \mathrm{loc}}\left(R^{3}\right), \\
\int_{\left|x_{1}-x_{1}^{\prime}\right| \leqq 1}\left|V_{i j}\left(\left|x_{1}\right|\right)\right|^{2} d x_{1} \rightarrow 0 \text { if }\left|x_{1}^{\prime}\right| \rightarrow \infty
\end{gathered}
$$

(that is $\left.V_{i j}\left(x_{1}\right) \in Q\left(R^{3}\right)[9]\right)$ and that

$$
t V_{i j}(t) \in \mathscr{L}_{1}\left(R_{+}^{1}\right) ;
$$

by virtue of [7] and condition (2.2) $V\left(x_{1}\right)=-\left|V_{i j}\left(x_{1}\right)\right| \in \mathscr{F}=\left\{V\left(x_{1}\right) \mid V\left(x_{1}\right) \in Q, \forall \varepsilon>0\right.$ the discrete spectrum of the operator $-\varepsilon \Delta_{1}+V\left(x_{1}\right)$ is finite in $\left.\mathscr{L}_{2}\left(R^{3}\right)\right\}$. Since $V\left(x_{1}\right) \in Q$ the operator $H_{0}$ with domain $C_{0}^{\infty}$ is essentially self-adjoint in $\mathscr{L}_{2}\left(R_{0}\right)$. With a slight abuse of notation we denote the self-adjoint extension of this operator by $H_{0}$.

Furthermore, given any invariant for $H_{0}$, we denote by $H_{0}^{\sigma}$ the restriction of the operator $H_{0}$ to the symmetry subspace $B^{\sigma} \subseteq \mathscr{L}_{2}\left(R_{0}\right)$ of the given symmetry $\sigma$. Then we denote for operator $H_{0}^{\sigma}$ the discrete spectrum as $\mathscr{I}_{d}\left(H_{0}^{\sigma}\right)$, the infinitely-multiple point spectrum as $\mathscr{I}_{p \infty}\left(H_{0}^{\sigma}\right)$, the greatest lower bound of the essential spectrum as $\mu^{\sigma}$.

We suppose everywhere $\mu^{\sigma}=0 .^{2}$

As spaces $B^{\sigma}$ we shall usually take spaces of the functions which are transformed according to the multiplying irreducible representations of Hamiltonian's $H_{0}$ symmetry group. The spaces $B^{\sigma}$, used in the article, will be constructed by projectors on the space of functions being transformed

i) according to the representation of the weight $l$ of the group $\mathrm{O}^{+}(3)$ - the projector $P^{(l)} l=0,1, \ldots$,

ii) according to the representation of the type $\alpha_{i}$ of the group $S_{3}$ - the projector $P^{\alpha_{i}} i=1,2$; here $\alpha_{1}=(1,1,1)$ is the type of the antisymmetric representation $S_{3} ; \alpha_{2}=(2,1)$ is the type of two-dimensional representation $S_{3}$,

iii) according to the antisymmetric representation of the group $S_{2}-$ the projector $P^{\alpha_{1}}\left(S_{2}\right)$.

First we consider the system $Z_{1}$ of three identical particles.

2 The case $\mu^{\sigma}<0$ was investigated earlier $[8,9,14,15]$ 
Theorem 2.1. Let $B^{\sigma}=P^{\alpha_{1}} \mathscr{L}_{2}\left(R_{0}\right)$. Then the spectrum $\mathscr{I}_{d}\left(H_{0}^{\sigma}\right)$ is finite and $0 \notin \mathscr{I}_{p \infty}\left(H_{0}^{\sigma}\right)$.

Theorem 2.2. Let $B^{\sigma}=P^{\alpha_{2}} P^{(0)} \mathscr{L}_{2}\left(R_{0}\right)$. Then the spectrum $\mathscr{I}_{d}\left(H_{0}^{\sigma}\right)$ is finite and $0 \notin \mathscr{I}_{p \infty}\left(H_{0}^{\sigma}\right)$.

Suppose now that the particles $i$ and $j$ in $Z_{1}$ are identical, and that the third particle may or may not be identical to these two. Let $S_{2}$ be the permutation group of particles $i$ and $j$.

Theorem 2.3. Let $B^{\sigma}=P^{\alpha_{1}}\left(S_{2}\right) P^{(0)} \mathscr{L}_{2}\left(R_{0}\right)$. Then the spectrum $\mathscr{I}_{d}\left(H_{0}^{\sigma}\right)$ is finite and $0 \notin \mathscr{I}_{p \infty}\left(H_{0}^{\sigma}\right)$.

The next theorems do not require the presence of identical particles in the system. Let $l \geqq 0$ be any integer number.

Theorem 2.4. Let

$$
B_{l}=\left\{\psi(x) \mid \psi(x) \in \mathscr{L}_{2}\left(R_{0}\right), \psi(-x)=(-1)^{l+1} \psi(x)\right\}, \quad B^{\sigma}=P^{(l)} B_{l} .
$$

Then the spectrum $\mathscr{I}_{d}\left(H_{0}^{\sigma}\right)$ is finite and $0 \notin \mathscr{I}_{p \infty}\left(H_{0}^{\sigma}\right)$.

Theorem 2.5. Let $B^{\sigma}=P^{(l)} \mathscr{L}_{2}\left(R_{0}\right)$. Then there exists a number $L>0$ such that $l>L$ the spectrum $\mathscr{I}_{d}\left(H_{0}^{\sigma}\right)$ is finite and $0 \notin \mathscr{I}_{p \infty}\left(H_{0}^{\sigma}\right)$.

\section{On Virtual Levels of Energy Operators for Two-Particle Systems}

Let us consider the operator

$$
h_{\varepsilon}=-\frac{1}{2}(1-\varepsilon) \Delta_{1}+V\left(\left|x_{1}\right|\right),
$$

where the function $V\left(\left|x_{1}\right|\right)$ satisfies the conditions (2.1), (2.2), $\varepsilon \in\left[0, \frac{1}{2}\right]$. The operator $h_{\varepsilon}$ with the domain $C_{0}^{\infty}\left(R^{3}\right)$ is essentially self-adjoint in $\mathscr{L}_{2}\left(R^{3}\right)$. We henceforth denote by $h_{\varepsilon}$ the self-adjoint extension of this operator. We denote its domain by $D\left(h_{\varepsilon}\right)$. By virtue of Corollary 2.9 [10] $D\left(h_{\varepsilon}\right)=D\left(h_{0}\right)$. According to [7] we denote by $\mathscr{L}_{2}^{(1)}$ the closure of space $W_{2}^{1}$ in the norm $|\psi|=\|\nabla \psi\|_{\mathscr{L}_{2}\left(R^{3}\right)}, \mathscr{L}_{2}^{(1)}$ is the complete Hilbert space with an inner product $[\varphi, \psi]=(\nabla \varphi, \nabla \psi)_{\mathscr{L}_{2}\left(R^{3}\right)^{3}}{ }^{3}$ Since $\forall b>0$

$$
\int_{\left|x_{1}\right| \leqq b}|\psi|^{2} d x_{1} \leqq b^{2} \int|\psi|^{2}\left|x_{1}\right|^{-2} d x_{1} \leqq 4 b^{2}|\psi|^{2},
$$

for $\psi \in W_{2}^{1}\left(R^{3}\right)$, so this inequality holds for $\varphi \in \mathscr{L}_{2}^{(1)}\left(R^{3}\right)$ too. Then $\mathscr{L}_{2}^{(1)}\left(R^{3}\right) \subset \mathscr{L}_{2, \text { loc }}\left(R^{3}\right)$ and therefore $\mathscr{L}_{2}^{(1)}\left(R^{3}\right) \subset W_{2}^{(1)}(\Omega)$ for any bounded region $\Omega$.

We remark that due to [7] the embeding operator $\mathscr{L}_{2}^{(1)}\left(R^{3}\right)$ in $\mathscr{L}_{2}\left(R^{3} ;|V|\right)$ is compact, that is

$$
\int|V|\left|\psi_{m}-\psi_{0}\right|^{2} d x_{1} \rightarrow 0 \quad \text { if } \quad \psi_{m} \rightarrow \psi_{0} \quad \text { at } \quad \mathscr{L}_{2}^{(1)}\left(R^{3}\right) .
$$

Let $e$ be an arbitrary set of non-negative integers $l^{\prime}$. Let $P(e)=\sum_{l^{\prime} \in e} P^{\left(l^{\prime}\right)}$, and let $h_{\varepsilon}(e)$ be the restriction of operator $h_{\varepsilon}$ to $P(e) D\left(h_{\varepsilon}\right)$. The operator $P(e)$ is a projector in $\mathscr{L}_{2}\left(R^{3}\right)$. It is easy to see that $P(e)$ is a projector in $W_{2}^{1}\left(R^{3}\right)$ too and hence it can be extended in $\mathscr{L}_{2}^{(1)}\left(R^{3}\right)$ as a projector. Let $\bar{B}(e)=P(e) \mathscr{L}_{2}^{(1)}\left(R^{3}\right)$.

3 Here and everywhere $(\nabla \varphi, \nabla \psi)_{\mathscr{L}_{2}\left(R^{3}\right)}=\int(\nabla \varphi, \nabla \psi)_{R^{3}} d \Omega$ 
Definition 3.1. We shall say the non-negative operator $h_{0}(e)$ has the virtual level if the negative spectrum of $h_{\varepsilon}(e)$ is not empty for any $\varepsilon \in\left(0, \frac{1}{2}\right)$.

Theorem 3.1. Suppose $0 \notin e$, and that the operator $h_{0}(e) \geqq 0$ has a virtual level. Then

i) the number $\lambda_{0}=0$ is an eigenvalue of the operator $h_{0}(e)$,

ii) the eigenspace $\mathscr{W}$ corresponding to the number $\lambda_{0}=0$ is in $P^{\left(l_{0}\right)} \mathscr{L}_{2}\left(R^{3}\right)$ and $\operatorname{dim} \mathscr{W}=2 l_{0}+1$, where $l_{0}=\min _{l^{\prime} \in e} l^{\prime}$,

iii) $\exists \delta>0$ such that $\left(h_{\delta} \psi, \psi\right)>0, \forall \psi \in D\left(h_{0}(e)\right),[\psi, u]=0$ for all $u \in \mathscr{W}$.

Remark. If $0 \in e$ and the operator $h_{0}(e)$ has a virtual level, then due to [5] the number 0 cannot be an eigenvalue of the operator $h_{0}(e)$ as distinct from the case $0 \notin e$. Just this distinction generates the principal difference in the structure of the discrete spectrum of Hamiltonians of the 3-particle systems depending on the fact whether the states with $l=0$ are permitted or not.

Proof. At first we shall prove

assertion $A$ : if $V(|x|)$ satisfies the theorem conditions and the function $T_{0}$ from $\mathscr{L}_{2}^{(1)}\left(R_{N}^{1}\right)$ is the generalized solution on $R_{N}^{14}$ of the equation

$$
T^{\prime \prime}-l(l+1) \varrho^{-2} T(\varrho)-2 V(\varrho) T(\varrho)=0,
$$

then $T_{0} \in \mathscr{L}_{2}\left(R_{N}^{1}\right)$ for sufficiently large $N$ and

$$
T_{0}=c\left(g+K g+K^{2} g+\ldots\right),
$$

where $c$ is a constant, $g=\varrho^{-l}, K f=\int \mathscr{K}(\varrho, t) f(t) d t, \mathscr{K}(\varrho, t)=-\bar{V}(t) \varrho^{l+1} t^{-l}$ if $t \geqq \varrho$, $\mathscr{K}(\varrho, t)=-\bar{V}(t) t^{l+1} \varrho^{-l}$ if $t \in[N, \varrho], \bar{V}(t)=V(t) \cdot(2 l+1)^{-1}$.

We note that $\bar{V}(t) \in \mathscr{L}_{2 \text {, loc }}$ and the generalized solution of Eq. (3.4) is the solution of this equation almost everywhere on $R_{N}^{1}$ for $N>0$. By virtue of Sobolev's embedding theorem $T_{0} \in C^{1}\left(R_{N}^{1}\right)$ and $T_{0}^{\prime}$ is absolutely continuous function on $R_{N}^{1}$. It is easy to see that the relation holds for any $\varrho>N$

$$
T_{0}(\varrho)=\left(d_{1}+\int_{N}^{\varrho} \bar{V}(t) T_{0}(t) t^{-l} d t\right) \varrho^{l+1}+\left(d_{2}-\int_{N}^{\varrho} \bar{V}(t) T_{0}(t) t^{l+1} d t\right) \varrho^{-l}
$$

where $d_{i}$ are constants depending on $T_{0}(N), T_{0}^{\prime}(N)$. Since $T_{0}^{\prime}(\varrho) \in \mathscr{L}_{2}\left(R_{N}^{1}\right)$ then

$$
d_{1}+\int_{N}^{\infty} \bar{V}(t) T_{0}(t) t^{-l} d t=0
$$

therefore the function $\tilde{T}=T_{0} d_{2}^{-1} 5$ is a solution of the equation

$$
\tilde{T}(\varrho)=g(\varrho)+\int_{N}^{\infty} \mathscr{K}(\varrho, t) \tilde{T}(t) d t .
$$

For the proof of assertion $A$ it is sufficient to show the function

$$
\tilde{T}_{0}=g+K g+K^{2} g+\ldots
$$

is in $\mathscr{L}_{2}\left(R_{N}^{1}\right)$ and that it is the unique solution in $\mathscr{L}_{2}^{(1)}$ of Eq. (3.6).

$4 \quad R_{N}^{1}=\left\{t \mid t \in R_{+}^{1}, t \geqq N\right\}, \mathscr{L}_{2}^{(1)}\left(R_{N}^{1}\right)$ is a closure $W_{2}^{1}\left(R_{N}^{1}\right)$ by the norm $|\psi|=\left\|\frac{\partial}{\partial t} \psi(t)\right\| \mathscr{L}_{2}\left(R_{N}^{1}\right)$

5 The number $N$ may be taken always so that $d_{2} \neq 0$ 
By virtue of the properties of the function $V(t)$ and because $\varepsilon>0$, one can choose the number $N$ so large that $|K g|<\varepsilon|g|$. From this and the fact that $g \in \mathscr{L}_{2}\left(R_{N}^{1}\right)$ for $N>0$, it follows that the series (3.7) is convergent and that $\tilde{T}_{0} \in \mathscr{L}_{2}\left(R_{N}^{1}\right)$. The operator $K$ can be applied to series (3.7) term by term, and therefore, $K \tilde{T}_{0}=\tilde{T}_{0}-g$ so that $\tilde{T}_{0}$ is the solution of (3.6). It is verified directly that $\tilde{T}_{0} \in \mathscr{L}_{2}^{(1)}\left(R_{N}^{1}\right)$.

Let us prove that the constructed solution of Eq. (3.6) is the unique one in $\mathscr{L}_{2}^{(1)}\left(R_{N}^{1}\right)$. For this it is sufficient to check that the homogeneous equation $f=K f$ has in $\mathscr{L}_{2}^{(1)}\left(R_{N}^{1}\right)$ only a trivial solution if the number $N$ is sufficiently large. Let $f(\varrho) \in \mathscr{L}_{2}^{(1)}\left(R_{N}^{1}\right)$, obviously,

$$
|f(\varrho)| \leqq|f(N)|+\left|\int_{N}^{\varrho} f^{\prime}(t) d t\right| \leqq|f(N)|+c \varrho^{1 / 2} .
$$

Therefore, the function $f_{1}(\varrho)=f(\varrho) \cdot \varrho^{-1 / 2}$ is bounded in $R_{N}^{1}$. If $f=K f$ and $f \in \mathscr{L}_{2}^{(1)}\left(R_{N}^{1}\right)$ then

$$
\begin{aligned}
\left|f_{1}(\varrho)\right| & \leqq \sup _{t \in R_{N}^{1}}\left\{\left|f_{1}(t)\right|\right\} \cdot \int_{N}^{\varrho}|\mathscr{K}(\varrho, t)| t^{1 / 2} \varrho^{-1 / 2} d t \\
& \leqq 0,5 \sup _{t \in R_{N}^{1}}\left\{\left|f_{1}(t)\right|\right\}
\end{aligned}
$$

when $N$ is large enough. It follows $f_{1}(t)=f(t)=0$ for $t \in R_{N}^{1}$ if $N$ is large enough. The assertion $A$ is proved.

Set

$$
\bar{W}^{l}=\left\{\psi(x) \mid \psi(x) \in \bar{B}^{l}=P^{(l)} \mathscr{L}_{2}^{(1)}\left(R^{3}\right), h_{0} \psi(x)=0 \text { in a generalized sense }\right\} .
$$

Let us prove

assertion $B$ : if under the condition of the theorem $\bar{W}^{l} \neq \emptyset$ for some $l>0$ then

i) the subspace $\bar{W}^{l}$ belongs to the domain of definition of the operator $h_{0}$ and is the eigenspace of the operator $h_{0}$ corresponding to the number $\lambda=0$;

ii) every function $\psi(x)$ from $\bar{W}^{l}$ has the form

$$
\psi(x)=\sum_{m=-l}^{l} c_{m} Y_{l m}(\theta, \varphi) R(|x|),
$$

where the function $R(|x|)$ is independent of $\psi(x) \in \bar{W}^{l}, c_{m}$ are constants depending on $\psi(x) ; Y_{l m}$ are spherical functions;

iii) $\operatorname{dim} \bar{W}^{l}=(2 l+1)$.

Let $\psi(x) \in \bar{W}^{l}$. Then $h_{0} \psi(x) \equiv-\frac{1}{2} \Delta_{1} \psi(x)+V \psi(x)=0$.

Since $P^{(l)} \psi(x)=\psi(x)$ we have

$$
\psi(x)=\sum_{m=-l}^{l} R_{l m}(\varrho) Y_{l m}(\theta, \varphi),
$$

where $\varrho=|x|, \theta, \varphi$ are the angular coordinates of $x$.

It is easy to see the function $T_{m}(\varrho)=R_{l m}(\varrho) \cdot \varrho$ is the generalized solution in $\mathscr{L}_{2}^{(1)}\left(R_{N}^{1}\right)$ for $N>0$ of the equation

$$
T_{m}^{\prime \prime}-l(l+1) \varrho^{-2} T_{m}(\varrho)-2 V(\varrho) T_{m}(\varrho)=0 .
$$


According to assertion $A$ the function $T_{m}(\varrho) \in \mathscr{L}_{2}\left(R_{N}^{1}\right)$ when $N$ is sufficiently large. Furthermore, $\psi(x) \in \mathscr{L}_{2, \text { loc }}\left(R^{3}\right)$. That is why $\psi(x) \in \mathscr{L}_{2}\left(R^{3}\right)$. Since the operator $h_{0}$ is self-adjoint and $\left(\psi(x), h_{0} \varphi(x)\right)=0$ for any function $\varphi(x) \in C_{0}^{\infty}\left(R^{3}\right)$ then $\psi(x) \in D\left(h_{0}\right)$ and $h_{0} \psi(x)=0$. Statement i) from assertion $B$ is proved.

Let us prove the statement ii). According to assertion $A$ and by virtue of the expansion (3.9) with $|x|>N$ the equality (3.8) holds if we set $R(|x|)=\tilde{T}_{0}(|x|)|x|^{-1}$ for $|x|>N$, where $\tilde{T}_{0}(|x|)$ is the sum of the series (3.7).

Since two different eigenfunctions of the operator $h_{0}$ which belong to $\bar{W}^{l}$ cannot coincide for $|x|>N$, one can define a function $R(|x|)$ so that the relation (3.8) holds for all $\psi(x) \in \bar{W}^{l}$. Statement ii) is proved. Statement iii) results from ii). Let us prove

assertion $C$ : if under conditions of Theorem $3.1 \bar{W}^{l} \neq \emptyset$ for some $l \in e$, then $l=l_{0}$. To prove this suppose $l>l_{0}$. Then evidently, $l \geqq 1$ and due to assertion $\mathrm{B}$ there is such a function $R(|x|)$ such that

$$
\psi(x)=\sum_{m=-l}^{l} c_{m} Y_{l m} R(|x|)
$$

for all $\psi(x) \in \bar{W}^{l}$. Further, we consider the function

$$
\tilde{\psi}(x)=Y_{l 0}(\theta, \varphi) R(|x|) .
$$

It is obvious that $\tilde{\psi}(x) \in \bar{W}^{l}$. Set $\bar{\psi}(x)=Y_{l_{0}, 0} R(|x|)$. Then

$$
\left(h_{0} \bar{\psi}(x), \bar{\psi}(x)\right)=\left(h_{0} \tilde{\psi}(x), \tilde{\psi}(x)\right)+\left[l_{0}\left(l_{0}+1\right)-l(l+1)\right] \cdot \int_{0}^{\infty}|R(t)|^{2} d t .
$$

Since $\left(h_{0} \tilde{\psi}(x), \tilde{\psi}(x)\right)=0, \quad\left(h_{0} \bar{\psi}(x), \bar{\psi}(x)\right) \geqq 0$ then $l_{0}\left(l_{0}+1\right)-l(l+1) \leqq 0$ and consequently $l=l_{0}$. Assertion $C$ is proved.

Let us begin immediately the proof of the theorem. Write $\mathscr{L}_{0}=\{\psi(x)|\psi \in \bar{B}(e),| \psi \mid=1\}$. Due to $\inf h_{\varepsilon}(e)<0$ with $\varepsilon>0$ and $h_{0}(e) \geqq 0$ it is easy to see that

$$
\inf _{\psi \in \mathscr{L}_{0}}(V \psi, \psi)=-\frac{1}{2}
$$

Let $\psi_{m}$ be a minimizing sequence for the functional $(V \psi, \psi)$ in $\mathscr{L}_{0}$. We choose from $\psi_{m}$ a sequence which is weakly convergent in $\mathscr{L}_{2}^{(1)}$. Abusing notation, we denote this subsequence by $\psi_{m}$. We denote the limit function as $u_{0}$. Evidently, $u_{0} \in \bar{B}(e)$ and $\left|u_{0}\right| \leqq \lim \inf \left|\psi_{m}\right|=1$.

By virtue of (3.11)

$$
\left(V u_{0}, u_{0}\right)=\lim _{m \rightarrow \infty}\left(V \psi_{m}, \psi_{m}\right)=-\frac{1}{2}
$$

Further, $\left|u_{0}\right|>0$ because the equality $\left|u_{0}\right|=0$ contradicts (3.12). We set $q=\left|u_{0}\right|$ It follows that $0<q \leqq 1$. Since $\tilde{u}_{0}=u_{0} q^{-1} \in \mathscr{L}_{0}$ then $\left(V \tilde{u}_{0}, \tilde{u}_{0}\right)=q^{-2}\left(V u_{0}, u_{0}\right)=-q^{-2} \frac{1}{2}$ $\geqq-\frac{1}{2}$ and consequently $q=1$. Here $\left|u_{0}\right|=1, u_{0} \in \mathscr{L}_{0}$ and $u_{0}$ realizes $\inf (V \psi, \psi)$ in $\mathscr{L}_{0}$.

By a common way we check that $u_{0}$ is a generalized solution of the equation

$$
h_{0} u_{0} \equiv-\frac{1}{2} \Delta_{1} u_{0}+V u_{0}=0 \text {. }
$$


Due to assertions B, C the function $u_{0} \in \bar{W}^{l_{0}}$ and statements i), ii) of the Theorem 3.1 hold. Now we shall prove statement iii) of the theorem. Let $u_{i}$, $i=0,1, \ldots, 2 l_{0}$ be an arbitrary basis in $\mathscr{W}$,

$$
\mathscr{L}_{1}=\left\{\psi(x) \mid \psi(x) \in \mathscr{L}_{0},\left[\psi, u_{i}\right]=0 \quad i=0,1, \ldots, 2 l_{0}\right\}
$$

In order to prove iii) it is sufficient to check the inequality

$$
\beta \equiv \inf _{\psi \in \mathscr{L}_{1}}(V \psi, \psi)>-\frac{1}{2} .
$$

Suppose it were not correct, and let $\beta=-\frac{1}{2}$. From any minimizing sequence $\psi_{m}$ for the functional $(V \psi, \psi)$ in $\mathscr{L}_{1}$ we choose a subsequence which converges weakly in $\mathscr{L}_{2}^{(1)}$. Let $\bar{u}$ be the limit function. As for $u_{0}$ one can verify $\bar{u} \in \mathscr{L}_{0}$ and $h_{0} \bar{u}=0$. By virtue of assertions $B, C$ we have $\bar{u} \in \mathscr{W}$ but it is impossible, because the function $\bar{u}$ being the weak limit of functions from $\mathscr{L}_{1}$ satisfies the conditions $\left[\bar{u}, u_{i}\right]=0$ $i=0,1, \ldots, 2 l_{0}$. Thus, $\bar{u} \notin \mathscr{W}$ and $\beta>-\frac{1}{2}$.

Theorem 3.1 is proved.

\section{Proofs of Theorems $2.1-2.5$}

Let $Z_{2}=\left(C_{1}, C_{2}\right)$ be an arbitrary breaking of the system $Z_{1}$ into two nonempty subsystems $C_{1}, C_{2}, C_{1} \cap C_{2}=\emptyset$. For definiteness we suppose $C_{1}$ always consists of two elements. We write

$$
R_{0}\left(Z_{2}\right)=\left\{x \mid x \in R_{0}, \sum_{i \in C_{1}} m_{i} x_{i}=0, C_{1} \in Z_{2}, x_{j}=0 j \notin C_{1}\right\}
$$

$R_{c}\left(Z_{2}\right)=R_{0} \ominus R_{0}\left(Z_{2}\right)^{6}, P_{c}\left(Z_{2}\right)$, and $P_{0}\left(Z_{2}\right)$ are the projectors in $R_{0}$ on $R_{c}\left(Z_{2}\right)$ and $R_{0}\left(Z_{2}\right)$.

We denote the operators $\nabla$ and $\Delta$ invariantly defined in $R_{0}\left(Z_{2}\right)$ and $R_{c}\left(Z_{2}\right)$ as $\nabla_{0}\left(Z_{2}\right), \Delta_{0}\left(Z_{2}\right)$ and $\nabla_{c}\left(Z_{2}\right), \Delta_{c}\left(Z_{2}\right)$, respectively. Let

$$
h_{0}\left(Z_{2}\right)=-\frac{1}{2} \Delta_{0}\left(Z_{2}\right)+V_{i j}\left(\left|x_{i j}\right|\right) \quad(i j) \in C_{1},
$$

$G\left(Z_{2}\right)$ be the group of operator $h_{0}\left(Z_{2}\right)$ symmetry, $\tilde{\sigma}=\tilde{\sigma}\left(Z_{2}\right)$ be the types of irreducible representations of group $G\left(Z_{2}\right), P^{\tilde{\sigma}}$ be the projector in $\mathscr{L}_{2}\left(R_{0}\left(Z_{2}\right)\right)$ on the subspace of functions being transformed according to representation of the type $\tilde{\sigma}$.

As in [6] (see also $[10$, p. 133]) we define the concept inducing $(\prec)$ of the symmetry $\tilde{\sigma}$ by the symmetry $\sigma$ and set

$$
B\left(\sigma ; Z_{2}\right)=\bigoplus_{\tilde{\sigma} \prec \sigma} P^{\tilde{\sigma}} \mathscr{L}_{2}\left(R_{0}\left(Z_{2}\right)\right)
$$

The restrictions of the operators $\Delta_{0}\left(Z_{2}\right)$ and $h_{0}\left(Z_{2}\right)$ to $B\left(\sigma ; Z_{2}\right)$ will be denoted by $\Delta_{0}\left(\sigma ; Z_{2}\right)$ and $h_{0}\left(\sigma ; Z_{2}\right)$, respectively. Let

$$
q\left(Z_{2}\right)=P_{0}\left(Z_{2}\right) x, \xi\left(Z_{2}\right)=P_{c}\left(Z_{2}\right) x, x \in R_{0} .
$$

$6 \Theta$ in the sense of inner product $(., .)_{1}$ 
For any $\beta>0, N>0$ we set

$$
\begin{aligned}
K\left(Z_{2} ; \beta\right) & =\left\{\left.x\left|x \in R_{0},\right| q\left(Z_{2}\right)\right|_{1} \leqq \beta\left|\xi\left(Z_{2}\right)\right|_{1}\right\}^{7}, \\
S(N) & =\left\{\left.x\left|x \in R_{0},\right| x\right|_{1}<N\right\} .
\end{aligned}
$$

Proof of Theorem 2.1. As in [11] to prove the theorem it is sufficient to show that for all $\psi \in D\left(H_{0}^{\sigma}\right)$ with $\operatorname{supp}\{\psi\}$ outside $S(N)$ the inequality

$$
L[\psi]=\left(H_{0} \psi, \psi\right)-\varepsilon\left\|\psi|x|_{1}^{-1}\right\|^{2}>0
$$

holds if $N$ is large and $\varepsilon>0$ is small. Evidently, the last inequality is satisfied if

$$
L_{1}[\psi] \equiv 0,5\left\|\left|\nabla_{0} \psi\right|\right\|^{2}+\sum_{i, j=1, i<j}^{3}\left(V_{i j} \psi, \psi\right)-\varepsilon\left\|\psi|x|_{1}^{-1}\right\|^{2}>0
$$

for all $\psi \in W_{2}^{1}\left(R_{0}\right) \cap B^{\sigma}, \operatorname{supp}\{\psi\} \cap S(N)=\emptyset$. Let $Z_{2}$ be any breaking, and let $q=q\left(Z_{2}\right)$ and $\xi=\xi\left(Z_{2}\right)$ be the coordinates in $R_{0}$. Under the conditions of the theorem the function $\tilde{\psi}(q, \xi) \equiv \psi(x) x \in R_{0}$ is odd relative to the changing of $q \leftrightarrow-q$ with an arbitrary fixed $\xi\left(Z_{2}\right)$. That is why the space $B\left(\sigma, Z_{2}\right)$ consists of odd functions and therefore $P^{(0)} B\left(\sigma ; Z_{2}\right)=\emptyset, \quad P^{(1)} B\left(\sigma ; Z_{2}\right) \neq \emptyset$. We suppose the operator $h_{0}\left(\sigma ; Z_{2}\right)$ has a virtual level ${ }^{8}$. By virtue of Theorem 3.1 and since $P^{(1)} B\left(\sigma ; Z_{2}\right) \neq \emptyset$ the number $\lambda=0$ is an eigenvalue of the operator $h_{0}\left(\sigma ; Z_{2}\right)$; the eigenspace $\mathscr{W}$ corresponding to $\lambda=0$ is in $P^{(1)} B\left(\sigma ; Z_{2}\right)$ and $\operatorname{dim} \mathscr{W}=3$; let $\varphi_{i}^{(0)}\left(q\left(Z_{2}\right)\right)_{i=1,2,3}$ be an orthonormal basis of $\mathscr{W}$. Let $\beta>0$ be so small that ${ }^{9}$

$$
K\left(Z_{2}^{\prime \prime} ; \beta\right) \cap K\left(Z_{2}^{\prime} ; \beta\right) \subset S(N) \text { if } Z_{2}^{\prime} \neq Z_{2}^{\prime \prime}
$$

For every $Z_{2}$ and $\varepsilon>0, \beta>0$ we find numbers $\alpha>0, N>0$ and functions $u_{Z_{2}}, v_{Z_{2}}$ according to Lemma $5.2^{10}$ with $\varphi_{i}=\varphi_{i}^{(0)}\left(q\left(Z_{2}\right)\right)$. Write $\mathscr{V}=\left(1-\sum_{Z_{2}} u_{Z_{2}}^{2}\right)^{1 / 2}$,
$\psi_{Z_{2}}=\psi u_{Z_{2}}, \bar{\psi}=\psi \mathscr{V}$. Then due to Lemma 5.2

where

$$
L_{1}[\psi] \geqq \sum_{Z_{2}} L_{2}\left[\psi_{Z_{2}}\right]+L_{3}[\bar{\psi}]
$$

$$
\begin{gathered}
L_{2}\left[\psi_{Z_{2}}\right]=\frac{1}{2}\left\|\left|\nabla_{0} \psi_{Z_{2}}\right|\right\|^{2}+\sum_{i, j=1, i<j}^{3}\left(V_{i j} \psi_{Z_{2}}, \psi_{Z_{2}}\right) \\
-4 \varepsilon\left\|\left|\nabla_{0}\left(Z_{2}\right) g\right|\right\|^{2}-\frac{1}{2} \sum_{k=1}^{3} \varepsilon\left\|\left|\nabla_{C}\left(Z_{2}\right) f_{k}\right|\right\|^{2}-\frac{1}{2} \varepsilon\left\|\psi_{Z_{2}}|x|_{1}^{-1}\right\|^{2}, \\
f_{k}=\left(\psi_{Z_{2}}, \Delta_{0}\left(Z_{2}\right) \varphi_{k}^{(0)}(q)\right)_{R_{0}\left(Z_{2}\right)}\left\|\left|\nabla_{0}\left(Z_{2}\right) \varphi_{k}^{(0)}\right|\right\|^{-2}, \\
g_{Z_{2}}=\psi_{Z_{2}}+\sum_{k=1}^{3} f_{k}\left(\xi\left(Z_{2}\right)\right) \varphi_{k}^{(0)}\left(q\left(Z_{2}\right)\right) \\
L_{3}[\bar{\psi}]=L_{1}[\bar{\psi}]-\varepsilon\left\|\bar{\psi}|x|_{1}^{-1}\right\|^{2} .
\end{gathered}
$$

7 For $y \in R_{0}|y|_{1}=(y, y)_{1}^{1 / 2}$

8 If the operator $h_{0}\left(\sigma ; Z_{2}\right)$ has no virtual level, then operators $h_{0}\left(\sigma ; Z_{2}^{\prime}\right)$ have no virtual levels for all $Z_{2}^{\prime}$ as a consequence of the identity of particles, in this case the finiteness $\mathscr{S}_{d}\left(H_{0}^{\sigma}\right)$ is proved in [11] (the finiteness of $\mathscr{S}_{d}\left(H_{0}\right)$ has been proved earlier in $\left.[15,16]\right)$

9 The fulfillment of relation (4.1) with sufficiently small $\beta>0$ is proved in [11]

10 All auxiliary assertions and their proofs are given in Sect. 5 
As far as $V_{i j} \in \mathscr{F}$ and $\left|x_{i j}\right|>\gamma N$ for some $\gamma>0$ and all $i, j, i \neq j$ if $x \in \operatorname{supp}\{\psi\}$ then one can establish as in [11] that $L_{3}[\bar{\psi}]>0$. Let us prove $L_{2}\left[\psi_{Z_{2}}\right]>0$. Set $Z_{2}=\{(s, p),(t)\}$. Taking into account that $h_{0}\left(Z_{2}\right) \varphi_{i}^{(0)}=0$ we have

$$
\begin{aligned}
L_{2}\left[\psi_{Z_{2}}\right]= & \frac{1}{2}\left\|\left|\nabla_{0}\left(Z_{2}\right) g_{Z_{2}}\right|\right\|^{2}+\left(V_{s p} g_{Z_{2}}, g_{Z_{2}}\right) \\
& +\left(\left(V_{s t}\left(x_{s t}\right)+V_{p t}\left(x_{p t}\right)\right) \psi_{Z_{2}}, \psi_{Z_{2}}\right)-4 \varepsilon\left\|\left|\nabla_{0}\left(Z_{2}\right) g_{Z_{2}}\right|\right\|^{2} \\
& +\frac{1}{2}(1-4 \varepsilon)\left\|\left|\nabla_{c}\left(Z_{2}\right) \psi_{Z_{2}}\right|\right\|^{2}-\frac{1}{2} \varepsilon \sum_{k=1}^{3}\left\|\left|\nabla_{c}\left(Z_{2}\right) f_{k}\right|\right\|^{2} .
\end{aligned}
$$

We can choose functions $\varphi_{i}^{(0)} i=1,2,3$ belonging to different lines of irreducible representation of weight 1 of the group $O^{+}(3)$. Then

$$
\left(\varphi_{i}^{(0)}, \varphi_{j}^{(0)}\right)_{R_{0}\left(Z_{2}\right)}=\left(\varphi_{i}^{(0)}, \Delta_{0}\left(Z_{2}\right) \varphi_{j}^{(0)}\right)_{R_{0}\left(Z_{2}\right)}=0 \quad \text { if } \quad i \neq j
$$

and therefore $\left(g_{Z_{2}}, \Delta_{0}\left(Z_{2}\right) \varphi_{i}^{(0)}\right)_{R_{0}\left(Z_{2}\right)}=0$.

By virtue of Theorem 3.1 if $\varepsilon>0$ is sufficiently small

$$
\left(\frac{1}{2}-8 \varepsilon\right)\left\|\left|\nabla_{0}\left(Z_{2}\right) g_{Z_{2}}\right|\right\|^{2}+\left(V_{s p} g_{Z_{2}}, g_{Z_{2}}\right)>0 .
$$

Since $V_{i j} \in \mathscr{F}$ then for sufficiently large $N$

$$
\varepsilon\left\|\left|\nabla_{c}\left(Z_{2}\right) \psi_{Z_{2}}\right|\right\|^{2}+\left(\left(V_{s t}\left(x_{s t}\right)+V_{p t}\left(x_{p t}\right)\right) \psi_{Z_{2}}, \psi_{Z_{2}}\right)>0 .
$$

In consequence of (4.5) to prove the inequality $L_{2}\left[\psi_{Z_{2}}\right]>0$ we need only show that with a sufficiently small $\varepsilon>0$

Evidently

$$
(1-6 \varepsilon)\left\|\left|\nabla_{c}\left(Z_{2}\right) \psi_{Z_{2}}\right|\right\|^{2}-\varepsilon \sum_{k=1}^{3}\left\|\left|\nabla_{c}\left(Z_{2}\right) f_{k}\right|\right\|^{2}>0
$$

$$
\begin{aligned}
\left\|\left|\nabla_{c}\left(Z_{2}\right) \psi_{Z_{2}}\right|\right\|^{2}= & \left\|\left|\nabla_{c}\left(Z_{2}\right) g_{Z_{2}}\right|\right\|^{2}+\sum_{k=1}^{3}\left\|\left|\nabla_{c}\left(Z_{2}\right) f_{k}\right|\right\|^{2} \\
& +2 \operatorname{Re} \sum_{k=1}^{3}\left(\nabla_{c}\left(Z_{2}\right) f_{k} \varphi_{k}^{(0)}, \nabla_{c}\left(Z_{2}\right) g_{Z_{2}}\right) .
\end{aligned}
$$

Let $g^{i}$ and $f_{k}^{i}$ be $i^{\text {th }}$ components of three dimensional vectors $\nabla_{c}\left(Z_{2}\right) g_{Z_{2}}$ and $\nabla_{c}\left(Z_{2}\right) f_{k}$. Since $\left(g_{Z_{2}}, \Delta_{0}\left(Z_{2}\right) \varphi_{k}^{(0)}\right)_{R_{0}\left(Z_{2}\right)}=0 \quad k=1,2,3$ almost everywhere in $R_{c}\left(Z_{2}\right)$ then $\left(g^{i}, \Delta_{0}\left(Z_{2}\right) \varphi_{k}^{(0)} f_{k}^{i}\right)=0 i, k=1,2,3$. By virtue of Lemma 5.3

$$
\begin{aligned}
\left|\operatorname{Re} \sum_{k=1}^{3}\left(\nabla_{c}\left(Z_{2}\right) f_{k} \varphi_{k}^{(0)}, \nabla_{c}\left(Z_{2}\right) g_{Z_{2}}\right)\right| & \leqq\left|\sum_{i=1}^{3}\left(g^{i}, \sum_{k=1}^{3} f_{k}^{i} \varphi_{k}^{(0)}\right)\right| \\
& \leqq \sum_{k=1}^{3} \sum_{i=1}^{3}\left(1-\delta_{k}\right)\left\|g^{i}\right\|\left\|f_{k}^{i} \varphi_{k}^{(0)}\right\|,
\end{aligned}
$$

where $\delta_{k}=\left\|\nabla_{y} \varphi_{k}^{(0)}(y)\right\|^{4}\left\|\Delta_{y} \varphi_{k}^{(0)}(y)\right\|^{-2}$. Write $\delta=\min \delta_{k}$. Then

$$
\begin{aligned}
& \left|\operatorname{Re} \sum_{k=1}^{3}\left(\nabla_{c}\left(Z_{2}\right) f_{k} \varphi_{k}^{(0)}, \nabla_{c}\left(Z_{2}\right) g_{Z_{2}}\right)\right| \leqq(1-\delta) \sum_{k=1}^{3} \sum_{i=1}^{3}\left\|g^{i}\right\|\left\|f_{k}^{i} \varphi_{k}^{(0)}\right\| \\
& =(1-\delta) \sum_{i=1}^{3}\left\|g^{i}\right\|\left\|\sum_{k=1}^{3} f_{k}^{i} \varphi_{k}^{(0)}\right\| \leqq 0,5(1-\delta)\left\|\left|\nabla_{c}\left(Z_{2}\right) g_{Z_{2}}\right|\right\|^{2} \\
& \quad+0,5(1-\delta) \sum_{k=1}^{3}\left\|\left|\nabla_{c}\left(Z_{2}\right) f_{k}\right|\right\|^{2} .
\end{aligned}
$$


From the last inequality and from (4.7) the relation (4.6) results if $\varepsilon<(1-6 \varepsilon) \delta$. Theorem 2.1 is proved.

Proof of Theorem 2.3. Let us suppose for definiteness that in the condition of the theorem $S_{2}$ is a group of permutations of particles 1 and 2. Then for breaking $Z_{2}^{0}=\left(C_{1}, C_{2}\right)$ with $C_{1}=(1,2)$ we can repeat all considerations of the proof of Theorem 2.1 up to inequality (4.2). In this repetition $\mathscr{V}=\left(1-u_{Z_{2}^{0}}^{2}\right), \bar{\psi}=\psi \mathscr{V}$. We shall obtain

$$
L_{1}[\psi] \geqq L_{2}\left[\psi_{Z_{2}^{0}}\right]+L_{3}[\bar{\psi}],
$$

where $L_{2}\left[\psi_{Z_{2}^{0}}\right]$ and $L_{3}[\bar{\psi}]$ are defined by relations (4.3), (4.4). As in Theorem 2.1 one can prove that $L_{2}\left[\psi_{Z_{2}^{0}}\right]>0$. Let us estimate $L_{3}[\bar{\psi}]$. Write

$$
\begin{gathered}
\Omega_{1}=\left\{x\left|x \in R_{0},\right| x_{13}|>| x_{23} \mid\right\} ; \Omega_{2}=R_{0} / \Omega_{1}, \\
\chi_{i}(x)=1 x \in \Omega_{i}, \chi_{i}(x)=0 x \notin \Omega_{i}, \psi_{i}=\bar{\psi} \chi_{i} .
\end{gathered}
$$

By virtue of Lemma $5.4 \operatorname{Tr} \bar{\psi}=0$ if $\left|x_{13}\right|=\left|x_{23}\right|$, then $\psi_{i} \in W_{2}^{1}$ and

$$
L_{3}[\bar{\psi}]=L_{3}\left[\psi_{1}\right]+L_{3}\left[\psi_{2}\right] \text {. }
$$

Without any loss of generality we suppose $\left\|\psi_{i}\right\|>0 i=1,2$. Let us shown that $L_{3}\left[\psi_{i}\right]>0$ if $N$ is sufficiently large. Setting $Z_{(23)}=\{(23),(1)\}$, we have

$$
\begin{aligned}
L_{3}\left[\psi_{1}\right]= & 0,5\left\|\left|\nabla_{0}\left(Z_{(23)}\right) \psi_{1}\right|\right\|^{2}+\left(V_{23} \psi_{1}, \psi_{1}\right) \\
& +0,5\left\|\left|\nabla_{c}\left(Z_{(23)}\right) \psi_{1}\right|\right\|^{2}+\left(\left(V_{12}\left(x_{12}\right)+V_{13}\left(x_{13}\right)\right) \psi_{1}, \psi_{1}\right) \\
& -2 \varepsilon\left\|\psi_{1}|x|_{1}^{-1}\right\|^{2} .
\end{aligned}
$$

Since $\operatorname{supp}\left\{\psi_{1}\right\} \cap\left\{K\left(Z_{2}^{0} ; \alpha\right) \cup S(N)\right\}=0$ one can find a $\gamma>0$ such that $x \in \operatorname{supp}\{\bar{\psi}\}$, the relation $\left|x_{12}\right|>\gamma N$ holds and so $\left|x_{13}\right| \geqq 0,5 \gamma N$ if $x \in \operatorname{supp}\left\{\psi_{1}\right\}$. Therefore, and due to $V_{12}, V_{13} \in \mathscr{F} ; \nabla_{c}\left(Z_{(23)}\right)=\operatorname{const} \nabla_{x_{13}}=\operatorname{const} \nabla_{x_{12}}$ with the fixed $q\left(Z_{2}\right)$, then the inequality

$$
0,25\left\|\left|\nabla_{c}\left(Z_{(23)}\right) \psi_{1}\right|\right\|^{2}+\left(\left(V_{12}\left(x_{12}\right)+V_{13}\left(x_{13}\right)\right) \psi_{1}, \psi_{1}\right)>0
$$

is satisfied when $N$ is sufficiently large. By virtue of Courant's inequality

$$
8 \varepsilon\left\|\left|\nabla_{c}\left(Z_{(23)}\right) \psi_{1}\right|\right\|^{2}-2 \varepsilon\left\|\psi_{1}|x|_{1}^{-1}\right\|^{2}>0 .
$$

Finally since $\psi_{1} \in B\left(\sigma_{0} ; Z_{(23)}\right)$ with $B^{\sigma_{0}}=P^{(0)} \mathscr{L}_{2}\left(R_{0}\right)$ and since $h\left(\sigma_{0} ; Z_{(23)}\right) \geqq 0$ we have

$$
\frac{1}{2}\left\|\left|\nabla_{0}\left(Z_{(23)}\right) \psi_{1}\right|\right\|^{2}+\left(V_{23} \psi_{1}, \psi_{1}\right)>0 \text {. }
$$

From the last three inequalities and (4.9) the relation $L_{3}\left[\psi_{1}\right]>0$ follows. Analogously, we obtain $L_{3}\left[\psi_{2}\right]>0$. The theorem is proved.

Proof of Theorem 2.2. Let $B_{2}^{\sigma}$ be a subspace of $B^{\sigma}$ space which consists of functions belonging to the second line of two-dimensional representation of $S_{3}$ group. It is obvious that it is sufficient to prove the finiteness of the discrete spectrum $H_{0}$ only on $B_{2}^{\sigma}$. Since $\psi\left(x_{1}, x_{2}, x_{3}\right)=-\psi\left(x_{2}, x_{1}, x_{3}\right)$ for $\psi\left(x_{1}, x_{2}, x_{3}\right) \in B_{2}^{\sigma}$, $B_{2}^{\sigma}$ is a subspace of the space $P^{\alpha_{1}}\left(S_{1}\right) P^{(0)} \mathscr{L}_{2}\left(R_{0}\right)$ from Theorem 2.3. That is why the finiteness of discrete spectrum $H_{0}$ on $B_{2}^{\sigma}$ can be proved as well as the finiteness of $\mathscr{S}_{d}\left(H_{0}^{\sigma}\right)$ in Theorem 2.3. 
Proof of Theorem 2.4. The theorem is proved as Theorem 2.1 because by virtue of Lemma $3.2[12] P^{(0)}\left(Z_{2}\right) B\left(\sigma ; Z_{2}\right)=\emptyset$ for any breaking $Z_{2}$; here $P^{(0)}\left(Z_{2}\right)$ is a projector $P^{(0)}$ in space $\mathscr{L}_{2}\left(R_{0}\left(Z_{2}\right)\right)$.

Proof of Theorem 2.5. As in the proof of Theorem 2.1 we shall show that

$$
L_{1}[\psi]=\frac{1}{2}\left\|\left|\nabla_{0} \psi\right|\right\|^{2}+\sum_{i, j=1 ; i<j}^{3}\left(V_{i j} \psi, \psi\right)-\varepsilon\left\|\psi|x|_{1}^{-1}\right\|^{2}>0
$$

for any function $\psi \in W_{2}^{1}\left(R_{0}\right) \cap B^{\sigma}$ with supp $\{\psi\}$ outside of $S(N)$ if $N$ is sufficiently large and $\varepsilon>0$ is small. Suppose $\beta>0$ had been taken so small that

$$
K\left(Z_{2}^{\prime} ; \beta\right) \cap K\left(Z_{2}^{\prime \prime} ; \beta\right) \subset S(N) \text { if } Z_{2}^{\prime \prime} \neq Z_{2}^{\prime} \text {. }
$$

With these given $\beta>0, \varepsilon>0$ we shall construct functions $u_{Z_{2}}, v_{Z_{2}}$ according to Lemma 5.1 and set $g_{Z_{2}}=P^{(0)}\left(Z_{2}\right) \psi u_{Z_{2}}, \phi_{Z_{2}}=\psi u_{Z_{2}}-g_{Z_{2}}$. By virtue of Lemma 5.2

$$
L_{1}[\psi] \geqq \sum_{Z_{2}} L_{2}\left[\psi_{Z_{2}}\right]+L_{3}[\bar{\psi}],
$$

where $\psi_{Z_{2}}=\psi u_{Z_{2}}, \bar{\psi}=\psi \mathscr{V}, \mathscr{V}=\left(1-\sum_{Z_{2}} u_{Z_{2}}^{2}\right)^{1 / 2}$

$$
\begin{aligned}
L_{2}\left[\psi_{Z_{2}}\right]= & \frac{1}{2}\left\|\left|\nabla_{0} \psi_{Z_{2}}\right|\right\|^{2}+\sum_{i, j=1, i<j}^{3}\left(V_{i j} \psi_{Z_{2}}, \psi_{Z_{2}}\right)-\varepsilon\left\|\psi_{Z_{2}}|x|_{1}^{-1}\right\|^{2} \\
& -\varepsilon\left\|\left|\nabla_{0}\left(Z_{2}\right) \phi_{Z_{2}}\right|\right\|^{2}-\frac{1}{2} c\left\|g_{Z_{2}}\left|\xi\left(Z_{2}\right)\right|_{1}^{-1}\right\|^{2} ; \\
L_{3}[\bar{\psi}]= & \frac{1}{2}\left\|\left|\nabla_{0} \bar{\psi}\right|\right\|^{2}+\sum_{i, j=1, i<j}^{3}\left(V_{i j} \bar{\psi}, \bar{\psi}\right)-2 \varepsilon\left\|\bar{\psi}|x|_{1}^{-1}\right\|^{2} .
\end{aligned}
$$

As in Theorem 2.1 we verify inequality $L_{3}[\bar{\psi}]>0$. Now we shall estimate the functional $L_{2}\left[\psi_{Z_{2}}\right]$. Write $Z_{2}=\{(i, j),(k)\}, g=g_{Z_{2}}, \phi=\phi_{Z_{2}}$. Since $P^{(0)}\left(Z_{2}\right) g=g$, $P^{(0)}\left(Z_{2}\right) \phi=0$ then $\left(\nabla_{0}\left(Z_{2}\right) \phi, \nabla_{0}\left(Z_{2}\right) g\right)=0$ and $\left(V_{i j} \phi, g\right)=0$, therefore

$$
\begin{aligned}
0,5 & \left\|\left|\nabla_{0} \psi_{Z_{2}}\right|\right\|^{2}+\sum_{i, j=1, i<j}^{3}\left(V_{i j} \psi_{Z_{2}}, \psi_{Z_{2}}\right)=\frac{1}{2}\left\|\left|\nabla_{0}\left(Z_{2}\right) \psi_{Z_{2}}\right|\right\|^{2} \\
& +\left(\left(V_{i k}\left(x_{i k}\right)+V_{j k}\left(x_{j k}\right)\right) \psi_{Z_{2}}, \psi_{Z_{2}}\right)+0,5\left\|\left|\nabla_{0}\left(Z_{2}\right) g\right|\right\|^{2} \\
& +\left(V_{i j} g, g\right)+0,5\left\|\left|\nabla_{0}\left(Z_{2}\right) \phi\right|\right\|^{2}+\left(V_{i j} \phi, \phi\right) .
\end{aligned}
$$

We remark that for the symmetry $\sigma$ considered in the theorem, $P^{(0)}\left(Z_{2}\right) B\left(\sigma ; Z_{2}\right) \neq \emptyset$ is fulfilled. Consequently, due to assertion $C$ (Sect. 3) the operator $h_{0}\left(\sigma ; Z_{2}\right)$ cannot have virtual levels on the spaces $P^{(l)}\left(Z_{2}\right) B\left(\sigma ; Z_{2}\right)$ with $l>0$. That is why for some $\varepsilon_{1}>0$ independent of $\psi$ we have

$$
0,5\left\|\left|\nabla_{0}\left(Z_{2}\right) \phi\right|\right\|^{2}+\left(V_{i j} \phi, \phi\right) \geqq \varepsilon_{1}\left\|\left|\nabla_{0}\left(Z_{2}\right) \phi\right|\right\|^{2}
$$

and

$$
0,5\left\|\left|\nabla_{0}\left(Z_{2}\right) \psi_{Z_{2}}\right|\right\|^{2}+\left(V_{i j} \psi_{Z_{2}}, \psi_{Z_{2}}\right) \geqq \varepsilon_{1}\left\|\left|\nabla_{0}\left(Z_{2}\right) \phi\right|\right\|^{2} .
$$

If $N$ is sufficiently large then

$$
\varepsilon\left\|\left|\nabla_{c}\left(Z_{2}\right) \psi_{Z_{2}}\right|\right\|^{2}+\left(\left(V_{i k}\left(x_{i k}\right)+V_{j k}\left(x_{j k}\right)\right) \psi_{Z_{2}}, \psi_{Z_{2}}\right)>0,
$$


furthermore

Hence $L_{2}\left[\psi_{Z_{2}}\right]>0$ if

$$
4 \varepsilon\left\|\left|\nabla_{c}\left(Z_{2}\right) \psi_{Z_{2}}\right|\right\|^{2}-\varepsilon\left\|\psi_{Z_{2}}|x|_{1}^{-1}\right\|^{2}>0
$$

$$
(1-10 \varepsilon)\left\|\left|\nabla_{c}\left(Z_{2}\right) \psi_{Z_{2}}\right|\right\|^{2}-c\left\|g\left|\xi\left(Z_{2}\right)\right|_{1}^{-1}\right\|^{2}>0 .
$$

It is obvious $\left\|\left|\nabla_{c}\left(Z_{2}\right) \psi_{Z_{2}}\right|\right\|^{2}-\left\|\left|\nabla_{c}\left(Z_{2}\right) g\right|\right\|^{2}>0$. To estimate $\left\|\left|\nabla_{c}\left(Z_{2}\right) g\right|\right\|$ we remark the function $g\left(q\left(Z_{2}\right), \xi\left(Z_{2}\right)\right)$ with fixed $\xi\left(Z_{2}\right)$ is in $P^{(0)}\left(Z_{2}\right) \mathscr{L}\left(R_{0}\left(Z_{2}\right)\right)$ and since $g(q, \xi) \in P^{(l)} \mathscr{L}_{2}\left(R_{0}\right)$ then

$$
\left\|\left|\nabla_{c}\left(Z_{2}\right) g\right|\right\|^{2} \geqq l(l+1)\left\|g\left|\xi\left(Z_{2}\right)\right|_{1}^{-1}\right\|^{2} .
$$

Hence (4.12) follows if $(1-10 \varepsilon) L(L+1)>c$ and $l \geqq L$. The theorem is proved.

\section{Auxiliary Assertions}

Lemma 5.1. Given arbitrary numbers $\beta>0, \varepsilon>0$ for each breaking $Z_{2}$ into two systems, one may choose numbers $\alpha \in(0, \beta), \alpha_{1} \in(\alpha, \beta)$ and real functions $u_{Z_{2}}(x), v_{Z_{2}}(x)$ $x \in R_{0}$ with piecewise continuous derivatives for $|x|_{1}>0$ such that $u_{Z_{2}}=\left(1-v_{Z_{2}}^{2}\right)^{1 / 2}$, $v_{Z_{2}}=1$ if $x \notin K\left(Z_{2} ; \beta\right), v_{Z_{2}}=0$ if $x \in K\left(Z_{2} ; \alpha\right)$, and the next inequalities hold

$$
\left\{\left|\nabla_{0} u_{Z_{2}}\right|^{2}+\left|\nabla_{0} v_{Z_{2}}\right|^{2}\right\}\left|v_{Z_{2}}\right|^{-2}<\varepsilon|x|_{1}^{-2}
$$

if $x \in K\left(Z_{2} ; \beta\right) / K\left(Z_{2} ; \alpha_{1}\right),|x|_{1}>0$;

$$
\left\{\left|\nabla_{0} u_{Z_{2}}\right|^{2}+\left|\nabla_{0} v_{Z_{2}}\right|^{2}\right\}\left|u_{Z_{2}}\right|^{-2}<\varepsilon\left|q\left(Z_{2}\right)\right|_{1}^{-2}
$$

if $x \in K\left(Z_{2} ; \alpha_{1}\right) / K\left(Z_{2}, \alpha\right),|x|_{1}>0$.

Corollary. For any function $\psi \in W_{2}^{1}\left(R_{0}\right)$ with $\operatorname{supp}\{\psi\}$ outside $S(N) N>0$ the following inequality holds:

$$
\begin{aligned}
\left\|\left|\nabla_{0} \psi\right|\right\|^{2} \geqq & \left\|\left|\nabla_{0} \psi v_{Z_{2}}\right|\right\|^{2}+\left\|\left|\nabla_{0} \psi u_{Z_{2}}\right|\right\|^{2} \\
& -\varepsilon\left\|\psi v_{Z_{2}}|x|_{1}^{-1}\right\|^{2}-\varepsilon\left\|\psi u_{Z_{2}}\left|q\left(Z_{2}\right)\right|_{1}^{-1}\right\|^{2} .
\end{aligned}
$$

Proof. Let $v_{1}(t)$ be such a real $C^{1}\left(R_{+}^{1}\right)$ function such that

1. $v_{1}(t)=1$ if $t \geqq \beta, v_{1}(t)$ monotonicly increases for $t \in[0, \beta]$.

2. $v_{1}^{\prime}(t)\left(1-v_{1}^{2}(t)\right)^{1 / 2} \rightarrow 0$ if $t \rightarrow \beta-0$.

We choose a number $\alpha_{1}$ sufficiently close to $\beta$ and set

Then

$$
v_{Z_{2}}(x)=v_{1}(t) \quad \text { if } \quad t \geqq \alpha_{1}, t=\left|q\left(Z_{2}\right)\right|_{1} \cdot\left|\xi\left(Z_{2}\right)\right|_{1}^{-1}, u_{Z_{2}}=\left(1-v_{Z_{2}}^{2}\right)^{1 / 2} .
$$

$$
\begin{aligned}
& \left\{\left|\nabla_{0} v_{Z_{2}}\right|^{2}+\left|\nabla_{0} u_{Z_{2}}\right|^{2}\right\}=\left\{1+\left(1-u_{Z_{2}}^{2}\right)^{-1}\right\}\left|\nabla_{0} v_{Z_{2}}\right|^{2}=\left\{1+\left(1-v_{Z_{2}}^{2}\right)^{-1}\right\} \\
& \cdot\left(1+\left|q\left(Z_{2}\right)\right|_{1}^{2}\left|\xi\left(Z_{2}\right)\right|_{1}^{-2}\right)\left|\xi\left(Z_{2}\right)\right|_{1}^{-2} v_{t}^{\prime 2} \leqq\left\{v_{t}^{\prime 2}+v_{t}^{\prime 2}\left(1-v^{2}\right)^{-1}\right\} \\
& \cdot\left(1+\beta^{2}\right) \alpha_{1}^{-2}\left(1+\alpha_{1}^{2}\right)|x|_{1}^{-2} .
\end{aligned}
$$

Since $v(t) \rightarrow 0, v_{t}^{\prime} \rightarrow 0, v_{t}^{\prime 2}\left(1-v^{2}\right) \rightarrow 0$ when $t \rightarrow \beta-0$ one can choose the number $\alpha_{1}$ so close to $\beta$ that

$$
\left\{v_{t}^{\prime 2}+v_{t}^{\prime 2}\left(1-v^{2}\right)^{-1}\right\}\left(1+\beta^{2}\right) \alpha_{1}^{-2}\left(1+\alpha_{1}^{2}\right)|v|^{-2}<\varepsilon
$$

and therefore inequality (5.1) is holds. 
Write $v_{1}\left(\alpha_{1}\right)=d_{1}, v_{2}(t)=d_{1} \cdot\left(\ln \left(\alpha_{1} / \alpha\right)\right)^{-1} \ln (t / \alpha)$. Let $v_{Z_{2}}(x)=v_{2}(t)$ if $t \in\left[\alpha, \alpha_{1}\right]$, $v_{Z_{2}}(x)=0$ if $t \in[0, \alpha], t=\left|q\left(Z_{2}\right)\right|_{1} \cdot\left|\xi\left(Z_{2}\right)\right|_{1}^{-1}$. At present functions $u_{Z_{2}}, v_{Z_{2}}$ are defined for all $x \in R_{0}$. It is obvious that to prove the lemma we must verify only (5.2) when $\alpha>0$ is sufficiently small. Since $\left|v_{2}(t)\right|<d_{1}<1$ if $t<\alpha_{1}$ then

$$
\begin{aligned}
&\left\{\left|\nabla_{0} u_{Z_{2}}\right|^{2}+\left|\nabla_{0} v_{Z_{2}}\right|^{2}\right\}\left|u_{Z_{2}}\right|^{-2}=\left|\nabla_{0} v_{Z_{2}}\right|^{2}\left\{1+\left(1-v_{Z_{2}}^{2}\right)^{-1}\right\} u_{Z_{2}}^{-2} \\
&<\left|\nabla_{0} v_{Z_{2}}\right|^{2}\left\{1+\left(1-d_{1}^{2}\right)^{-1}\right\}\left(1-d_{1}^{2}\right)^{-1} . \\
&\left|\nabla_{0} v_{Z_{2}}\right|^{2}=\left|\nabla_{0}\left(Z_{2}\right) v_{Z_{2}}\right|^{2}+\left|\nabla_{c}\left(Z_{2}\right) v_{Z_{2}}\right|^{2}=\left|\xi\left(Z_{2}\right)\right|_{1}^{-2} \\
& \cdot\left(1+\left|q\left(Z_{2}\right)\right|_{1}^{2}\left|\xi\left(Z_{2}\right)\right|_{1}^{-2}\right) v_{t}^{\prime 2} \leqq\left(1+\alpha_{1}^{2}\right)\left|\xi\left(Z_{2}\right)\right|_{1}^{-2} v_{t}^{\prime 2} .
\end{aligned}
$$

So far as

$$
v_{t}^{\prime 2}=d_{1}^{2}\left(\ln \left(\alpha_{1} / \alpha\right)\right)^{-2}\left|\xi\left(Z_{2}\right)\right|_{1}^{2}\left|q\left(Z_{2}\right)\right|_{1}^{-2},
$$

then (5.2) follows from (5.4), (5.5) if $\alpha$ was taken so small that

$$
\left(\ln \left(\alpha_{1} / \alpha\right)\right)^{2} \varepsilon>\left(1+\alpha_{1}^{2}\right)\left\{1+\left(1-d_{1}^{2}\right)^{-1}\right\} d_{1}^{2} \cdot\left(1-d_{1}^{2}\right) .
$$

Lemma 5.1 is proved.

Lemma 5.2. Choose $\varepsilon>0, \beta>0$ arbitrarily, and let $Z_{2}$ be any breaking into two subsystems. Construct functions $v_{Z_{2}}$ and $u_{Z_{2}}$ according to Lemma 5.1 with the chosen values of $\varepsilon$ and $\beta$. Let $\psi, g, \phi \in W_{2}^{1}\left(R_{0}\right)$ be any functions with supports outside $S(N)$, $g=\psi u_{Z_{2}}-\phi$.

Then: i) one can choose $c>0$ independent of $\psi, g, \phi$ such that

$$
\begin{aligned}
\left\|\left|\nabla_{0} \psi\right|\right\|^{2} \geqq & \left\|\left|\nabla_{0} \psi v_{Z_{2}}\right|\right\|^{2}+\left\|\left|\nabla_{0} \psi u_{Z_{2}}\right|\right\|^{2}-\varepsilon\left\|\psi v_{Z_{2}}|x|_{1}^{-1}\right\|_{K\left(Z_{2}, \beta\right)}^{2} \\
& -8 \varepsilon\left\|\left|\nabla_{0}\left(Z_{2}\right) g\right|\right\|^{-2}-c\left\|\phi\left|\xi\left(Z_{2}\right)\right|_{1}^{-1}\right\|_{K\left(Z_{2} ; \beta\right) \mid K\left(Z_{2} ; \alpha\right)}^{2}
\end{aligned} ;
$$

ii) if $\phi=\sum_{i=1}^{m} \varphi_{i}\left(q\left(Z_{2}\right)\right) f_{i}\left(\xi\left(Z_{2}\right)\right)$, where $\varphi_{i}(q) i=1, \ldots, m$ are fixed functions from $W_{2}^{1}\left(R_{0}\left(Z_{2}\right)\right)$ and $f_{i}(\xi)$ are any functions from $W_{2}^{1}\left(R_{c}\left(Z_{2}\right)\right)$, then one can choose $N$ independent of $\psi, f_{i} i=1, \ldots, m$ such that

$$
\begin{aligned}
\left\|\left|\nabla_{0} \psi\right|\right\|^{2} \geqq & \left\|\left|\nabla_{0} \psi u_{Z_{2}}\right|\right\|^{2}+\left\|\left|\nabla_{0} \psi v_{Z_{2}}\right|\right\|^{2}-8 \varepsilon\left\|\left|\nabla_{0}\left(Z_{2}\right) g\right|\right\|^{2} \\
& -\varepsilon\left\|\psi v_{Z_{2}}|x|_{1}^{-1}\right\|_{K\left(Z_{2} ; \beta\right)}^{2}-\sum_{i=1}^{m} \varepsilon\left\|\left|\xi\left(Z_{2}\right)\right|_{1}^{-1} f_{i}(\xi)\right\|^{2} .
\end{aligned}
$$

Proof. From relations (5.1), (5.2) it follows that to prove (5.6) it is sufficient to verify the inequality

$$
\begin{aligned}
& \left\|\psi u_{Z_{2}}\left|q\left(Z_{2}\right)\right|_{1}^{-1}\right\|_{K\left(Z_{2} ; \beta\right) / K\left(Z_{2} ; \alpha\right)}^{2} \leqq 8\left\|\left|\nabla_{0}\left(Z_{2}\right) g\right|\right\|^{2} \\
& \quad+c\left\|\phi\left|\xi\left(Z_{2}\right)\right|_{1}^{-1}\right\|_{K\left(Z_{2} ; \beta\right) / K\left(Z_{2} ; \alpha\right)}^{2} .
\end{aligned}
$$

Since $\left|\psi u_{Z_{2}}\right|^{2} \leqq 2|g|^{2}+2|\phi|^{2}$ and

$$
8\left\|\left|\nabla_{0}\left(Z_{2}\right) g\right|\right\|^{2}-2\left\|g\left|q\left(Z_{2}\right)\right|_{1}^{-1}\right\|^{2} \geqq 0,
$$

then the inequality (5.8) will result from the relation

$$
2\left\|\phi\left|q\left(Z_{2}\right)\right|_{1}^{-1}\right\|_{K\left(Z_{2} ; \beta\right) / K\left(Z_{2} ; \alpha\right)}^{2} \leqq c\left\|\phi\left|\xi\left(Z_{2}\right)\right|_{1}^{-1}\right\|_{K\left(Z_{2} ; \beta\right) / K\left(Z_{2} ; \alpha\right)}^{2}
$$

that is valid if $c>2 \alpha^{-1}$ because $\left|q\left(Z_{2}\right)\right|_{1}>\alpha\left|\xi\left(Z_{2}\right)\right|_{1}$ for $r \notin K\left(Z_{2} ; \alpha\right)$. 
Let us prove (5.7). For this, due to (5.6) it is sufficient to prove that

$$
c\left\|\phi\left|\xi\left(Z_{2}\right)\right|_{1}^{-1}\right\|_{K\left(Z_{2} ; \beta\right) / K\left(Z_{2} ; \alpha\right)}^{2} \leqq \sum_{i=1}^{m} \varepsilon\left\|\left|\xi\left(Z_{2}\right)\right|_{1}^{-1} \cdot f_{i}\right\|^{2} .
$$

Evidently

$$
\left\|\phi\left|\xi\left(Z_{2}\right)\right|_{1}^{-1}\right\|_{K\left(Z_{2} ; \beta\right) / K\left(Z_{2} ; \alpha\right)}^{2} \leqq m \sum_{i=1}^{m}\left\|\left|\xi\left(Z_{2}\right)\right|_{1}^{-1} \varphi_{i} f_{i}\right\|_{K\left(Z_{2} ; \beta\right) / K\left(Z_{2} ; \alpha\right)}^{2}
$$

Since

$$
q\left(Z_{2}\right) \in \Omega_{N}=\left\{\left.q\left(Z_{2}\right)|| q\left(Z_{2}\right)\right|_{1}>N\left(1+\alpha^{-2}\right)^{1 / 2}\right\}
$$

if

$$
r \in \operatorname{supp}\left\{\varphi_{i}(q) f_{i}(\xi)\right\} \cap\left\{K\left(Z_{2} ; \beta\right) / K\left(Z_{2} ; \alpha\right)\right\}
$$

then $\int_{\Omega_{N}}\left|\varphi_{i}(q)\right|^{2} d q \rightarrow 0$ with $N \rightarrow \infty$; that is why relation (5.9) holds for sufficiently large $N$. The lemma is proved.

Lemma 5.3. Let $x \in R^{3}, \quad y \in R^{3}, \quad g(x, y) \in \mathscr{L}_{2}\left(R^{6}\right), \quad f(x) \in \mathscr{L}_{2}\left(R^{3}\right), \quad \varphi(y) \in D\left(\Delta_{y}\right)$, $\phi=\varphi(y) f(x)$ and $\left(g(x, y), \Delta_{y} \phi(x, y)\right)=0$. Then

$$
|(g(x, y), \phi(x, y))| \leqq(1-\delta)\|g\|\|\phi\|,
$$

where

$$
\delta=0,5\left\|\nabla_{y} \varphi\right\|^{4}\|\varphi\|^{-2}\left\|\Delta_{y} \varphi\right\|^{-2} .
$$

Proof. Let $F_{+}=g\|g\|^{-1}+\phi\|\phi\|^{-1}, F_{-}=g\|g\|^{-1}-\phi\|\phi\|^{-1}$. Since $\left(g, \Delta_{y} \phi\right)=0$, then $\left(\left(F_{\oplus)}(\overline{+}) \phi\|\phi\|^{-1}\right), \Delta_{y} \phi\right)=0$ and therefore $\left(F_{( \pm)}, \Delta_{y} \phi\right)=(\mp)\left\|\nabla_{y} \phi\right\|^{2}\|\phi\|^{-1}$. Hence

$$
\left\|\nabla_{y} \phi\right\|^{2}\|\phi\|^{-1} \leqq\left\|F_{\text {由 }}\right\|\left\|\Delta_{y} \phi\right\|,
$$

that is $\left\|F_{( \pm)}\right\| \geqq\left\|\nabla_{y} \phi\right\|^{2}\|\phi\|^{-1}\left\|\Delta_{y} \phi\right\|^{-1} \equiv(2 \delta)^{1 / 2}$. Substituting $F_{+}$and $F_{-}$and squaring both parts of inequality we obtain

$$
\begin{aligned}
& (g, \phi)\|g\|^{-1}\|\phi\|^{-1}=-\frac{1}{2}\left\|F_{-}\right\|^{2}+1 \leqq 1-\delta, \\
& (g, \phi)\|g\|^{-1}\|\phi\|^{-1}=-1+\frac{1}{2}\left\|F_{+}\right\|^{2} \geqq-1+\delta .
\end{aligned}
$$

The lemma is proved.

Lemma 5.4. Let particles $i$ and $j$ be identical, and suppose

$$
\psi(x)=\psi\left(x_{1}, x_{2}, x_{3}\right) \in C_{0}\left(R_{0}\right)
$$

is antisymmetrical under the permutation $x_{i} \leftrightarrow x_{j}$ and $P^{(0)} \psi=\psi$. Then $\psi(x)=0$ if $\left|x_{k i}\right|=\left|x_{k j}\right| k \neq i, j$.

Proof. For definiteness let $i=1, j=2$. We find $x_{3}$ from condition $x \in R_{0}: m_{1} x_{1}$ $+m_{2} x_{2}+m_{3} x_{3}=0$ and set $\varphi\left(x_{1}, x_{2}\right) \equiv \psi\left(x_{1}, x_{2},-\tilde{m}^{-1}\left(x_{1}+x_{2}\right)\right) \equiv \psi\left(x_{1}, x_{2}, x_{3}\right)$, where $\tilde{m}=m_{3} m_{1}^{-1}$. It is obvious that $P^{(0)} \varphi=\varphi$ and $\varphi\left(x_{1}, x_{2}\right)=-\varphi\left(x_{2}, x_{1}\right)$. 
Expanding $\varphi\left(x_{1}, x_{2}\right)$ in spherical functions we shall obtain

$$
\varphi\left(x_{1}, x_{2}\right)=\sum a_{l_{1} m_{1}}^{l_{2} m_{2}}\left(\varrho_{1}, \varrho_{2}\right) Y_{l_{1} m_{1}}\left(\alpha_{1}\right) Y_{l_{2} m_{2}}\left(\alpha_{2}\right),
$$

where $\varrho_{i}=\left|x_{i}\right|, \alpha_{i}$ are angular coordinates of $x_{i}$. Since $P^{(0)} \varphi=\varphi$, then $a_{l_{1} m_{1}}^{l_{2} m_{2}}=0$ if $\left(l_{1}, m_{1}\right) \neq\left(l_{2},-m_{2}\right)$. Therefore

$$
\varphi=\sum_{l=0}^{\infty} \sum_{m=-l}^{l} a_{l m}\left(\varrho_{1}, \varrho_{2}\right) Y_{l_{1} m_{1}}\left(\alpha_{1}\right) Y_{l_{2} m_{2}}\left(\alpha_{2}\right),
$$

where $a_{l m}=a_{l, m}^{l,-m}$. For each $l$ the vector $\left\{a_{l m} m=-l, \ldots, l\right\}$ is colinear to the vector $B_{l m ; l,-m}^{o 0}$ of Clebsch-Gordan's coefficients. That is, why (see [13] p. 160) $a_{l m}\left(\varrho_{1}, \varrho_{2}\right)$ $=a_{l,-m}\left(\varrho_{1}, \varrho_{2}\right)$ and consequently

$$
\begin{aligned}
\varphi= & \sum_{l=0}^{\infty}\left[a_{l 0} Y_{l 0}\left(\alpha_{1}\right) Y_{l 0}\left(\alpha_{2}\right)+\sum_{m=1}^{l} a_{l m}\left(Y_{l m}\left(\alpha_{1}\right) Y_{l,-m}\left(\alpha_{2}\right)\right.\right. \\
& \left.\left.+Y_{l,-m}\left(\alpha_{1}\right) Y_{l m}\left(\alpha_{2}\right)\right)\right] .
\end{aligned}
$$

From this it follows that the function $\tilde{\varphi}\left(\varrho_{1}, \varrho_{2}, \alpha_{1}, \alpha_{2}\right) \equiv \varphi\left(x_{1}, x_{2}\right)$ is symmetrical under permutation $\alpha_{1} \leftrightarrow \alpha_{2}$ and therefore antisymmetrical under the permutation $\varrho_{1} \leftrightarrow \varrho_{2}$

By virtue of this fact $\tilde{\varphi}\left(\varrho, \varrho, \alpha_{1}, \alpha_{2}\right)=0$ for all $\alpha_{1}, \alpha_{2}$. But the equality $\left|x_{1}\right|=\left|x_{2}\right|$ is equivalent to equality $\left|x_{1}-x_{3}\right|=\left|x_{2}-x_{3}\right|$ with $\tilde{m} x_{3}=-\left(x_{1}+x_{2}\right)$. Thus, we have established that $\psi(x)=0$ if $\left|x_{13}\right|=\left|x_{23}\right|$. The lemma is proved.

Acknowledgement. We wish to thank Prof. G. Hagedorn for improving the English in our paper and Prof. B. Simon for his care of our manuscript.

\section{References}

1. Efimov, V.: Phys. Lett. B33, 563-564 (1970)

2. Yafaev, D.R.: Math. Sborn. 94, 567-593 (1974)

3. Ovchinnikov, Y.N., Sigal, I.M. : Ann. Phys. 123, 274-295 (1979)

4. Efimov, V.: Yadernaya Fizika 12, 1080-1091 (1970)

5. Yafaev, D.R.: Proceedings of Scientific Seminars. LOMI, 51, 203-216 (1975)

6. Sigalov, A.G., Sigal, I.M.: Theor. Math. Phys. 5, 73-94 (1973)

7. Birman, M.S. : Math. Sborn. 55, 125-174 (1961)

8. Yafaev, D.R.: Izv. AN SSSR (Ser. Math.) 40, 908-948 (1976)

9. Zhislin, G.M.: Theor. Math. Phys. 21, 60-73 (1974)

10. Antonets, M.A., Zhislin, G.M., Shereshevskij, I.A.: Appendix to the book of Jörgens, K., Weidmann, J., "Spectral properties of Hamiltonian operators", Moscow: Mir, 1976

11. Vugal'ter, S.A., Zhislin, G.M.: Rep. Math. Phys. (1981) (to appear)

12. Zhislin, G.M.: Izv. AN SSSR (Ser. Math.) 33, 590-649 (1969)

13. Gel'fand, I.M., Minlos, R.A., Shapiro, Z.Y.: Representation of the rotation and the Lorentz group. Moscow: Fizmatgiz 1958

14. Sigal, I.M.: Commun. Math. Phys. 48, 137-154 (1976)

15. Sigal, I.M.: Commun. Math. Phys. 48, 155-164 (1976)

16. Yafaev, D.R.: Theor. Math. Phys. 25, 189-195 (1975)

Communicated by B. Simon

Received July 22, 1981; in revised form May 20, 1982 
